\title{
Heterogeneous Intravascular Ultrasound Findings of Stent Thrombosis
}

\author{
Toru Morofuji, Shinji Inaba, Hiroe Aisu, Kayo Takahashi, Makoto Saito, Haruhiko Higashi, \\ Toyofumi Yoshii and Takumi Sumimoto
}

\begin{abstract}
Objective The underlying mechanisms of stent thrombosis are not completely understood.

Methods We experienced 12 definite stent thrombosis cases (1 early, 1 late, and 10 very late) at our hospital from July 2011 to April 2016 and evaluated the possible causes of stent thrombosis by intravascular ultrasound (IVUS).

Results Five different potential morphological causes of stent thrombosis (neoatherosclerosis, stent malapposition, stent fracture, edge dissection, and stent underexpansion) were detected by IVUS in 10 cases (83.3\%); in 1 of the remaining 2 cases, the discontinuation of antithrombotic drugs resulted in early stent thrombosis without abnormal IVUS findings. Of the 12 stent thrombosis cases, 4 occurred at a bare-metal stent (average time from stent implantation, 106 months); in all 12, significant neointimal hyperplasia was observed on IVUS, and 2 had plaque ruptures at an in-stent or proximal reference. Malapposed stent struts were observed in three very-late stent thromboses, and all of these underwent sirolimus-eluting stent implantation. Stent thrombosis due to mechanical (stent fracture) or procedure-related complications (edge dissection and stent underexpansion) was observed in three cases.

Conclusion In patients with stent thrombosis, heterogeneous findings were observed in IVUS. This IVUS case series illustrates the possible mechanisms of stent thrombosis.
\end{abstract}

Key words: stent thrombosis, intravascular ultrasound, bare-metal stent, drug-eluting stent

(Intern Med 56: 259-268, 2017)

(DOI: 10.2169/internalmedicine.56.7093)

\section{Introduction}

Stent implantation has been shown to reduce the rate of restenosis compared with balloon angioplasty alone; in particular, the use of drug-eluting stents has resulted in a dramatic reduction in the rate of neointimal formation over the last decade (1). However, concerns have been raised regarding stent thrombosis (2). Stent thrombosis is a rare but fatal complication of percutaneous coronary intervention (3-6). Therefore, the prevention of stent thrombosis is important in the clinical setting. Intravascular imaging and pathological studies have reported that various causes are associated with stent thrombosis, depending on stent types and time since implantation (7-9). Nevertheless, the underlying mechanisms of stent thrombosis are not completely understood. Accord- ingly, the aim of the present study was to investigate the possible causes of stent thrombosis using intravascular ultrasound (IVUS).

\section{Materials and Methods}

We retrospectively analyzed 601 consecutive patients who underwent percutaneous coronary intervention from July 2011 to April 2016 in Kitaishikai Hospital. The institutional review board approved the retrospective use of the patients' data. Definite stent thrombosis was diagnosed according to the Academic Research Consortium criteria (10). Early, late, and very-late stent thromboses were defined as follows: early stent thrombosis, 0-30 days; late stent thrombosis, 31 days-1 year; very-late stent thrombosis, $>1$ year post-stent implantation. 
Table 1. Mechanisms of Bare-metal Stent Failure in 8 Lesions by Intravascular Ultrasound.

\begin{tabular}{ccc}
\hline & Stent thrombosis $(+)$ & $\mathbf{( - )}$ \\
\hline Abnormal vascular response & $\mathbf{4}$ & $\mathbf{4}$ \\
-Neointimal hyperplasia & 4 & 4 \\
with plaque rupture & 2 & 0 \\
without plaque rupture & 2 & 4 \\
-Stent malapposition & 0 & 0 \\
Mechanical and procedure-related complications & $\mathbf{0}$ & $\mathbf{0}$ \\
\hline
\end{tabular}

Table 2. Mechanisms of Drug-eluting Stent Failure in 36 Lesions by Intravascular Ultrasound.

\begin{tabular}{ccc}
\hline & Stent thrombosis $(+)$ & $\mathbf{( - )}$ \\
\hline Abnormal vascular response & $\mathbf{3}$ & $\mathbf{2 0}$ \\
\hline -Neointimal hyperplasia & 0 & 20 \\
with plaque rupture & 0 & 0 \\
without plaque rupture & 0 & 20 \\
-Stent malapposition & 3 & 0 \\
Mechanical and procedure-related complications & $\mathbf{3}$ & $\mathbf{8}$ \\
-Stent underexpansion & 1 & 8 \\
-Others & 2 & 0 \\
Unkown cause & $\mathbf{2}$ & $\mathbf{0}$ \\
\hline
\end{tabular}

\section{Intravascular ultrasound}

IVUS imaging was performed after intracoronary administration of $0.1-0.2 \mathrm{mg}$ nitroglycerin using a commercially available IVUS system (iLab with 40-MHz Atlantis SR Pro catheters, Boston Scientific, Fremont, CA, USA; or VISIWAVE with 40-MHz Viewit catheters, Terumo, Tokyo, Japan) with automatic pullback at 0.5 or $1.0 \mathrm{~mm} / \mathrm{s}$. If necessary, at the operator's discretion, we used an aspiration catheter or small-sized balloon to open the occluded artery or severe stenosis before the IVUS examination. IVUS analyses were performed through the agreement of two independent cardiologists (TM and SI), who were blinded to the clinical data, stent type, and time of occurrence of the stent thrombosis. Regarding the definitions for the IVUS qualitative analysis, plaque rupture was defined as a cavity that communicated with the lumen with an overlying residual fibrous cap fragment (11); stent malapposition was defined as blood speckle behind the stent struts that did not overlie a side branch (12); and complete stent fracture was defined as complete separation of the stent into $\geq 2$ pieces separated by image slices with no visible stent struts (13).

The statistical analysis was performed using the StatView 5.0 software program (SAS Institute, Cary, NC, USA). Continuous variables were displayed as median values with the interquartile range (IQR; first and third) and compared between groups using the Mann Whitney $U$ test. Values of $\mathrm{p}<$ 0.05 were considered statistically significant.

\section{Results}

In the present study, stent failure which needed target lesion revascularization was seen in 45 lesions of 43 patients, which were treated with 36 drug-eluting stents (DESs), 8 bare-metal stents (BMSs), and 1 unknown stent. Of the lesions with stent failure, stent thrombosis occurred in 12 lesions $(26.7 \%$ per lesion) of 12 patients (27.9\% per patient). The mechanism of stent failure was able to be identified by IVUS in 43 lesions $(95.6 \%)$. In the BMSs, the mechanism of stent failure was only due to an abnormal vascular response (neointimal hyperplasia). Regarding the abnormal vascular response, stent malapposition was not observed in any of the cases of BMS failure. Plaque rupture, which is the most common cause of coronary thrombosis, was detected only in the lesions with stent thrombosis (Table 1). In contrast, heterogeneous IVUS findings were seen in the cases of DES failure, especially in the lesions with stent thrombosis, where an abnormal vascular response was observed in 20 lesions by IVUS. All 20 of these stent failures were due to neointimal hyperplasia without plaque rupture, while stent malapposition was not detected. In contrast, in the lesions with stent thrombosis, the mechanism of stent failure by an abnormal vascular response was only stent malapposition (Table 2).

Stent failure due to mechanical and procedure-related complication occurred earlier than those instances of failure due to abnormal vascular response (449 [IQR, 252-739] vs. $2,396$ [1,016-3,124] days, $\mathrm{p}=0.0011)$. Furthermore, stent failure in the DES-treated lesions occurred earlier than in the BMS-treated lesions (1,016 [IQR, 338-2,342] vs. 3,927 $[3,185-4,590]$ days, $p<0.0001)$. Even when the comparison was limited to the lesions with an abnormal vascular response, similar results were obtained (DES vs. BMS; 1,455 [IQR, 742-2,978] vs. 3,927 [3,185-4,590] days, $\mathrm{p}=0.0005)$.

The findings of the 12 patients with stent thrombosis are summarized in Table 3. In the present study, IVUS revealed various possible causes of stent thrombosis (neoatherosclerosis, stent malapposition, stent fracture, edge dissection, and 
Table 3. Findings in 12 Patients with Stent Thrombosis.

\begin{tabular}{|c|c|c|c|c|c|c|c|c|}
\hline Patient No. & Age/sex & $\begin{array}{c}\text { Clinical } \\
\text { presentation } \\
\text { at index } \\
\text { procedure } \\
\end{array}$ & $\begin{array}{l}\text { Clinical } \\
\text { presentation } \\
\text { at stent } \\
\text { thrombosis } \\
\end{array}$ & $\begin{array}{c}\text { Culprit } \\
\text { vessel }\end{array}$ & Antithrombotic drugs & Stent & $\begin{array}{c}\text { Duration from } \\
\text { stent implantation } \\
\text { (months) }\end{array}$ & $\begin{array}{l}\text { Possible cause of stent } \\
\text { thrombosis using IVUS }\end{array}$ \\
\hline \multicolumn{9}{|c|}{ Bare-metal stent } \\
\hline No.1 (Fig. 1) & $61 / \mathrm{M}$ & EAP & STEMI & RCA & Aspirin $100 \mathrm{mg}$ & BMS & 107 & \multirow{2}{*}{$\begin{array}{l}\text { Neointimal plaque rupture } \\
\text { Proximal reference plaque } \\
\text { rupture }\end{array}$} \\
\hline No.2 (Fig. 2) & $67 / \mathrm{M}$ & STEMI & STEMI & LAD & Aspirin $200 \mathrm{mg}$ & BMS & 96.8 & \\
\hline No.3 & $78 / \mathrm{M}$ & EAP & NSTEMI & RCA & $\begin{array}{l}\text { Aspirin } 200 \mathrm{mg} \\
\text { Clopidogrel } 75 \mathrm{mg}\end{array}$ & BMS & 125 & Neointimal hyperplasia \\
\hline No.4 & $64 / \mathrm{M}$ & STEMI & STEMI & RCA & Aspirin $81 \mathrm{mg}$ & BMS & 95.5 & Neointimal hyperplasia \\
\hline \multicolumn{9}{|c|}{$\underline{\text { Drug-eluting stent }}$} \\
\hline No.5 (Fig. 3) & $46 / \mathrm{M}$ & STEMI & STEMI & LAD & Aspirin $100 \mathrm{mg}$ & SES & 55.9 & $\begin{array}{l}\text { Malapposition due to positive } \\
\text { remodeling }\end{array}$ \\
\hline No.6 & $84 / \mathrm{M}$ & EAP & STEMI & LAD & Aspirin $100 \mathrm{mg}$ & SES & 101.4 & $\begin{array}{l}\text { Malapposition due to positive } \\
\text { remodeling }\end{array}$ \\
\hline No.7 & $68 / \mathrm{F}$ & unknown & STEMI & LAD & None & SES & 55 & Malapposition \\
\hline \multicolumn{9}{|c|}{ Mechanical and procedure-related complications } \\
\hline No.9 (Fig. 5) & $85 / \mathrm{F}$ & NSTEMI & NSTEMI & LCX & $\begin{array}{l}\text { Aspirin } 100 \mathrm{mg} \\
\text { Clopidogrel } 75 \mathrm{mg}\end{array}$ & CoCr-EES & 6.6 & Stent edge dissection \\
\hline No.10 (Fig. 6) & $87 / \mathrm{M}$ & EAP & STEMI & LAD & $\begin{array}{l}\text { Aspirin } 100 \mathrm{mg} \\
\text { Clopidogrel } 50 \mathrm{mg}\end{array}$ & SES & 47.5 & Stent underexpansion \\
\hline \multicolumn{9}{|c|}{ Unknown cause by IVUS } \\
\hline No.11 (Fig. 7) & $86 / \mathrm{M}$ & EAP & STEMI & LAD & None & PtCr-EES & 0.6 & None \\
\hline No.12 & $75 / F$ & EAP & STEMI & RCA & $\begin{array}{c}\text { Aspirin } 100 \mathrm{mg} \\
\text { Clopidogrel } 25 \mathrm{mg}\end{array}$ & PES & 49.6 & None \\
\hline
\end{tabular}

BMS: bare-metal stent, CoCr-EES: cobalt-chromium everolimus-eluting stent, EAP: effort angina pectoris, F: female, Fig: figure, IVUS: intravascular ultrasound, LAD: left anterior descending artery, LCX: left circumflex artery, M: male, NSTEMI: non-ST-segment elevation myocardial infarction, PES: paclitaxel-eluting stent, PtCr-EES: platinum-chromium everolimus-eluting stent, RCA: right coronary artery, SES: sirolimus-eluting stent, STEMI: ST-segment elevation myocardial infarction

stent underexpansion). Of the 12 definite stent thrombosis cases, we will present the 7 cases that illustrate the different causes of stent thrombosis.

\section{Case Nos. 1 and 2: Neoatherosclerosis (neointimal and proximal reference plaque rupture)}

\section{Case No. 1 (Fig. 1): Neointimal plaque rupture}

A 61-year-old man was implanted with two overlapping BMSs $(3.5 \times 23$ and $3.5 \times 13 \mathrm{~mm})$ in the proximal right coronary artery for effort angina pectoris. After 107 months, the patient was admitted to our hospital because of sudden chest pain. Emergency coronary angiography showed total occlusion proximal to the BMS site. After thrombus aspiration, IVUS was performed, and neointimal plaque rupture was observed.

\section{Case No. 2 (Fig. 2): Stent proximal reference plaque rupture}

A 67-year-old man was implanted with three BMSs (3.0x $12,3.0 \times 15$, and $3.5 \times 16 \mathrm{~mm})$ in the mid-left anterior descending coronary artery (LAD) for ST-segment elevation myocardial infarction. After 96.8 months, the patient was admitted to our hospital because of sustained chest discomfort. Emergency coronary angiography showed total occlusion within $5 \mathrm{~mm}$ of the site of the stent's proximal edge. After balloon angioplasty and thrombus aspiration, IVUS was performed, and stent proximal reference plaque rupture was observed.

\section{Case No. 5 (Fig. 3): Late-acquired stent malapposi- tion}

A 46-year-old man was implanted with a $3.0 \times 23-\mathrm{mm}$ sirolimus-eluting stent (SES) at the proximal portion of the LAD for ST-segment elevation myocardial infarction. After 55.9 months, the patient was admitted to our hospital because of sustained chest pain. Emergency coronary angiography revealed a total occlusion distal to the SES-implanted site. IVUS was performed after thrombus aspiration and balloon angioplasty. Stent malapposition was observed, while post-procedural IVUS images at the index intervention showed well-apposed stents.

\section{Case No. 8 (Fig. 4): Complete stent fracture}

An 89-year-old man was initially implanted with a 3.0x 28-mm SES in the mid-LAD for ST-segment elevation myocardial infarction. A second procedure was performed six months after the index SES placement because of lesion progression at the stent proximal site. In the second procedure, the patient was implanted with a $3.0 \times 18-\mathrm{mm}$ SES, which overlapped the old stent. At 67.6 months after the first procedure, the patient was admitted to our hospital because of sustained chest pain. Emergency coronary angiography revealed obvious stent fracture at the site of total occlusion near the overlapping site. IVUS was performed after thrombus aspiration and balloon angioplasty, confirming complete separate stent fracture with displacement.

\section{Case No. 9 (Fig. 5): Progression of stent edge dis- section}

An 85-year-old woman was implanted with a $2.25 \times 23$ - 

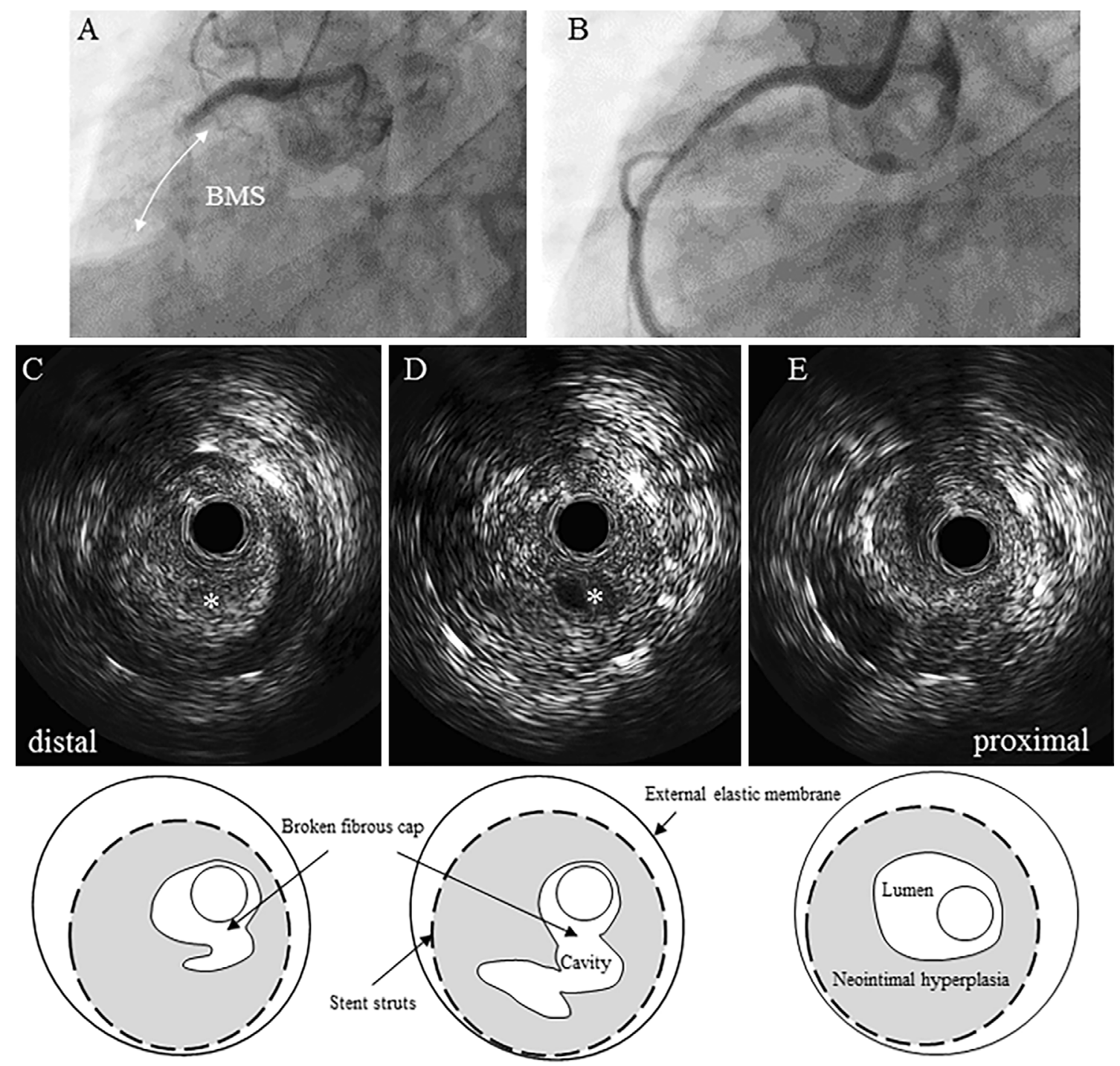

Figure 1. A: Coronary angiogram of the right coronary artery showing total occlusion at the proximal part of the bare-metal stent. B: Coronary angiogram after thrombus aspiration. C-E: Intravascular ultrasound images showing in-stent neointimal hyperplasia (C-E) with a ruptured cavity (D, asterisk).

mm cobalt-chromium everolimus-eluting stent (CoCr-EES) in the proximal left circumflex artery for non-ST-segment elevation myocardial infarction. After 6.6 months, the patient was admitted to our hospital because of resting angina with troponin elevation. Coronary angiography showed subtotal occlusion with thrombosis in myocardial infarction (TIMI) grade 2 flow from the stent proximal reference site. IVUS revealed edge dissection that was already observed at the index intervention. Proximal edge dissection at the index intervention reached the media, although there were no flowlimiting obstructions or significant abnormalities shown by angiography (TIMI 3 flow).

\section{Case No. 10 (Fig. 6): Chronic stent underexpansion}

An 87 -year-old man was implanted with a $2.5 \times 13-\mathrm{mm}$ SES in the proximal LAD for effort angina pectoris. After 47.5 months, the patient was admitted to our hospital because of sudden chest pain. Emergency coronary angiogra- phy revealed total occlusion at the SES-implanted site. After thrombus aspiration, IVUS was performed and revealed stent underexpansion (minimum stent area $[\mathrm{MSA}]=3.8 \mathrm{~mm}^{2}$ ). We confirmed that MSA at the index procedure was $4.0 \mathrm{~mm}^{2}$, indicating chronic stent underexpansion, not stent recoil.

\section{Case No. 11 (Fig. 7): Discontinuation of antithrom- botic drugs}

An 86-year-old man was successfully implanted with a 3.0 $\times 16-\mathrm{mm}$ platinum-chromium (PtCr) EES for effort angina pectoris. After discharge, the patient stopped taking antithrombotic drugs (clopidogrel and aspirin). Seven days after interruption of antiplatelet therapy (18 days after stenting), the patient was admitted to our hospital because of cardiogenic shock. Emergency coronary angiography showed thrombogenic occlusion at the stent site. IVUS was performed after thrombus aspiration and revealed thrombus-like appearances with no possible causes of stent thrombosis. 
A

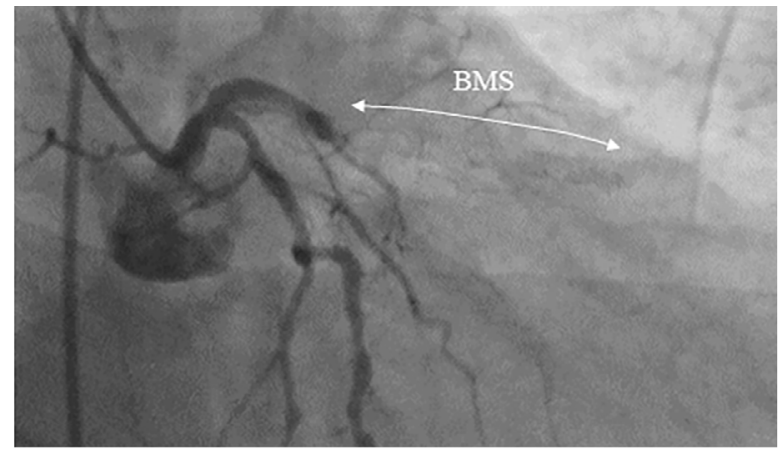

External elastic membrane
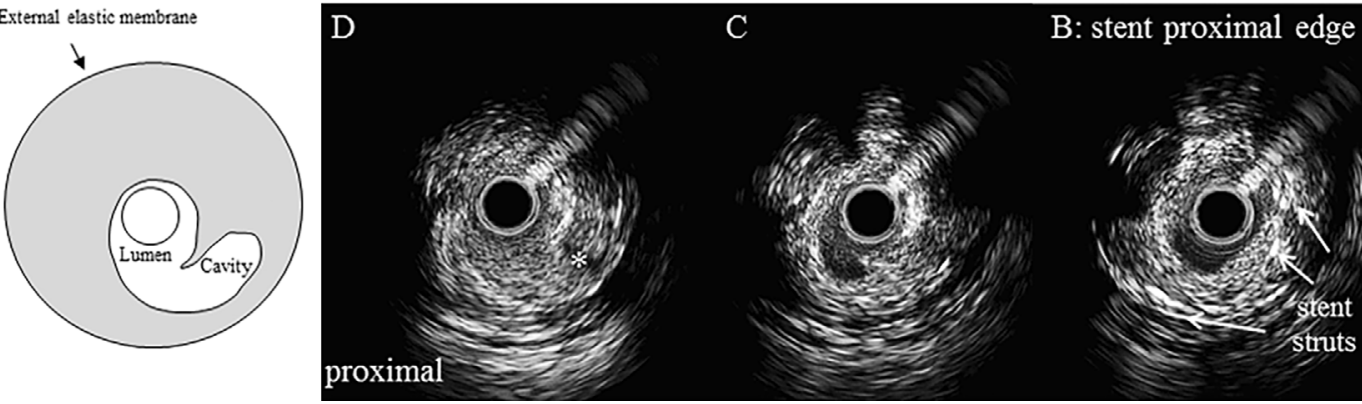

Figure 2. A: Coronary angiogram of the left anterior descending coronary artery showing total occlusion. B-D: Intravascular ultrasound images showing plaque rupture (asterisk) at the site of proximal reference adjacent to significant neointimal hyperplasia.

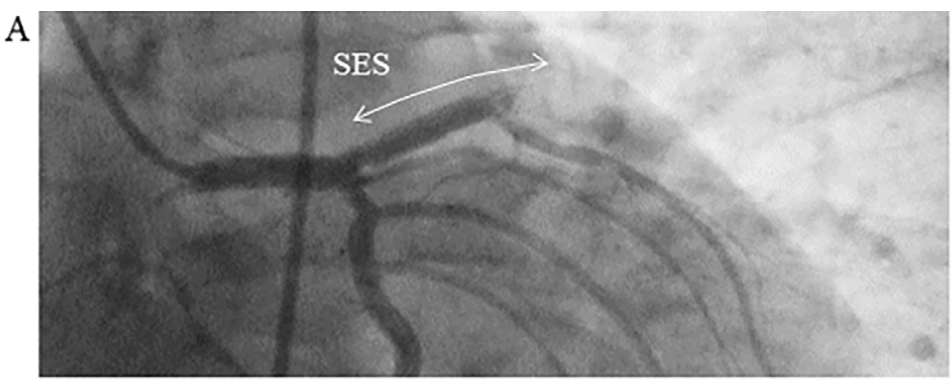

Baseline: post-procedure at index intervention

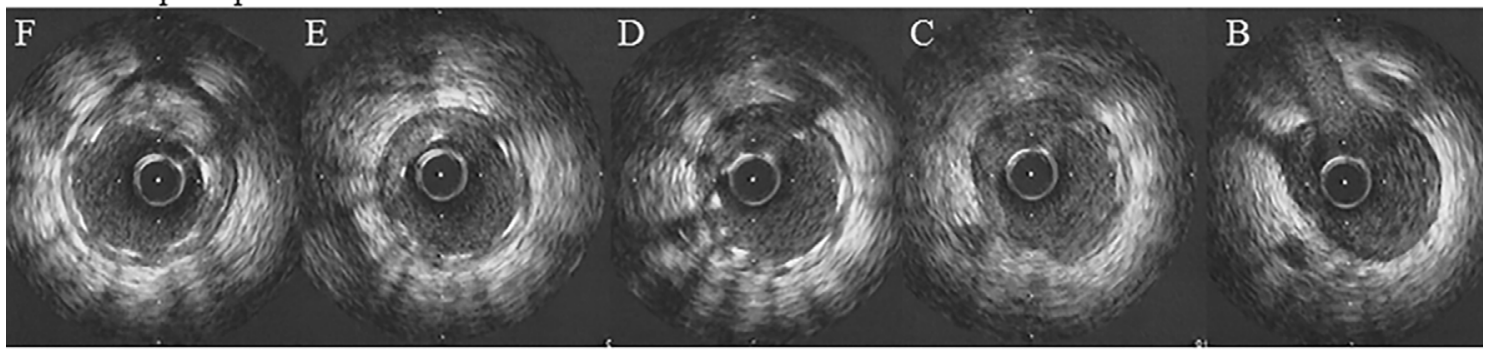

Stent thrombosis: 55.9 months after

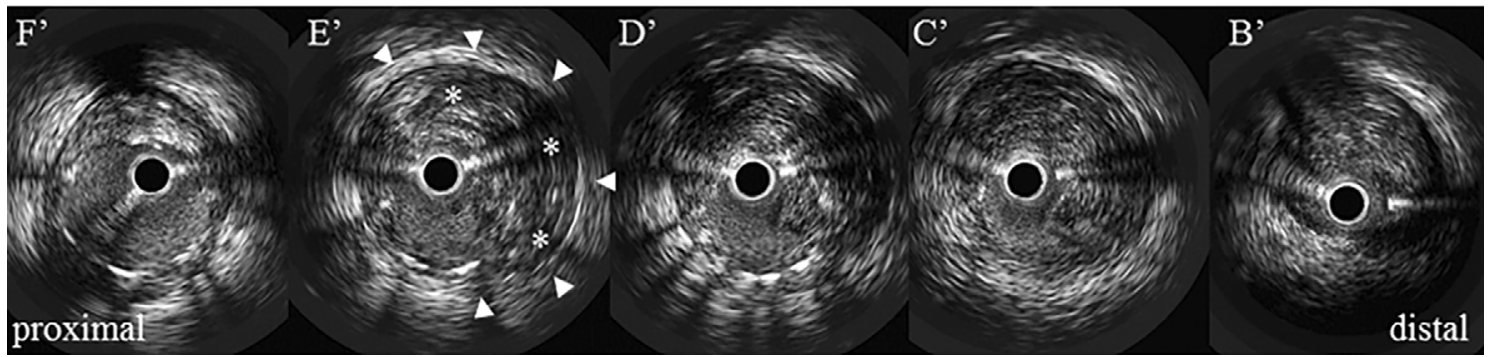

Figure 3. A: Coronary angiogram of the left anterior descending coronary artery showing total occlusion. B-F: Post-procedure intravascular ultrasound (IVUS) images at the index intervention showing no stent malapposition. B'-F': IVUS images at the stent thrombosis corresponding to the baseline IVUS images (B-F) showing stent malapposition (asterisk). The same location of the external elastic membrane cross-sectional area ( $E$ and $E$ ') was markedly enlarged, from 11.4 to $25.8 \mathbf{~ m m}^{2}$, suggesting development of late-acquired stent malapposition due to positive remodeling (white arrowheads). 

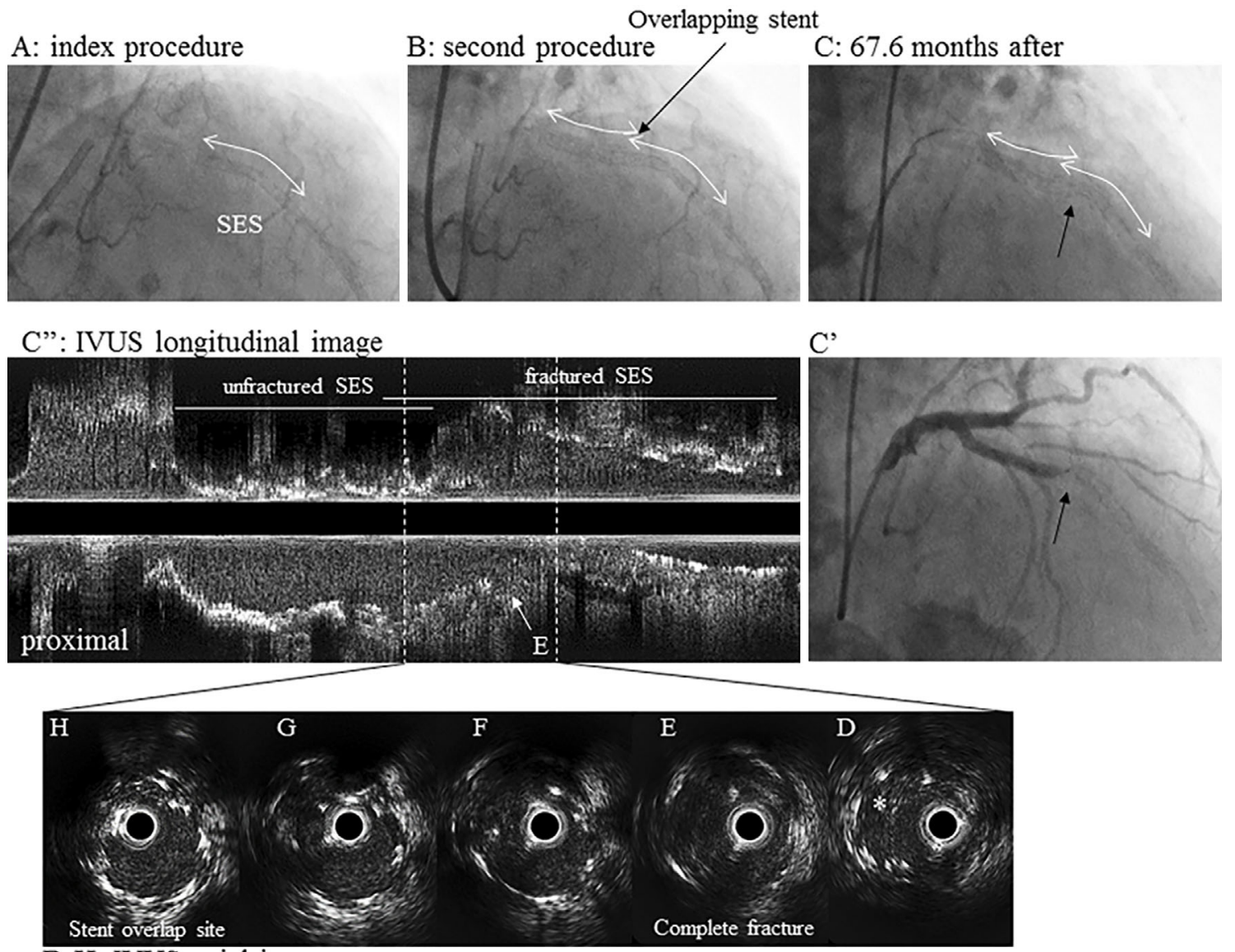

D-H: IVUS axial images

Figure 4. A: Coronary angiogram of the left anterior descending coronary artery without dye at the index procedure. B: Coronary angiogram without dye at the second procedure. $C-C^{\prime}$ : Coronary angiogram without $(C)$ and with dye $\left(C^{\prime}\right)$ at 67.6 months after the first procedure showing stent fracture (black arrow). C": Intravascular ultrasound (IVUS) longitudinal image confirming stent fracture (white arrow). D-H: IVUS axial images at fracture site corresponding to C'. (E) Complete separation of the single stent indicating complete stent fracture, $(\mathrm{H})$ double layered stent struts indicating stent overlap. *thrombus-like appearance.

\section{Discussion}

The main findings of the present study are as follows: (1) Of the lesions with stent failure, the frequency of stent thrombosis was $26.7 \%$, and stent thrombosis in $21.9 \%$ was due to abnormal vascular response and in $27.3 \%$ due to mechanical and procedure-related complications; (2) the timing of stent failure might depend on its mechanisms and stent types; and (3) heterogeneous IVUS findings were more commonly observed in the patients with DES failure with stent thrombosis than in those with BMS failure with stent thrombosis. Although only neointimal hyperplasia was found to be a mechanism of BMS failure, half of the lesions with stent thrombosis had plaque rupture, which can trigger thrombosis.

\section{Abnormal vascular response}

Pathological studies have revealed that the major cause of very-late stent thrombosis is an abnormal vascular response (i.e. hypersensitivity reaction, excessive fibrin deposition, and neoatherosclerosis) $(8,9)$. We experienced seven cases (Nos. 1-7) in which an abnormal vascular response (neoatherosclerosis and malapposition) may have been associated with the development of very-late stent thrombosis.

\section{Neoatherosclerosis}

This concept was proposed by Nakazawa et al. based on pathological observations (8). Their group named newly formed atherosclerotic changes within the neointimal tissue of stented segments "neoatherosclerosis." Although neoatherosclerosis can develop after both BMS and DES implantation, it has been reported more frequently and earlier with DESs than in BMSs. However, unstable lesions characterized as thin-cap fibroatheroma or plaque rupture are more common with BMSs than DESs $(8,9)$; this was confirmed by IVUS and optical coherence tomographic studies $(14,15)$ and may be due to the longer time from stent implantation with BMSs than DESs. In the present study, two neointimal plaque ruptures with BMSs were detected using IVUS, and 


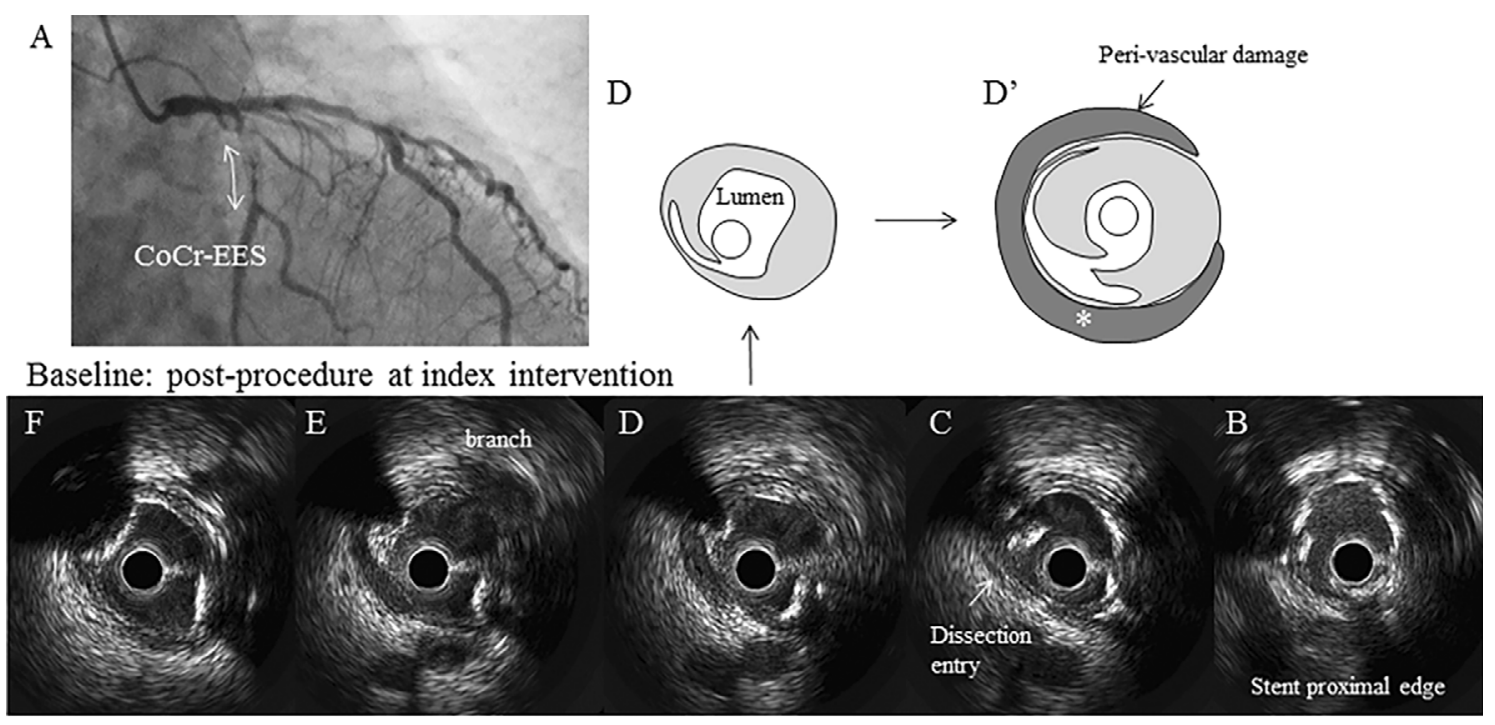

Stent thrombosis: 6.6 months after

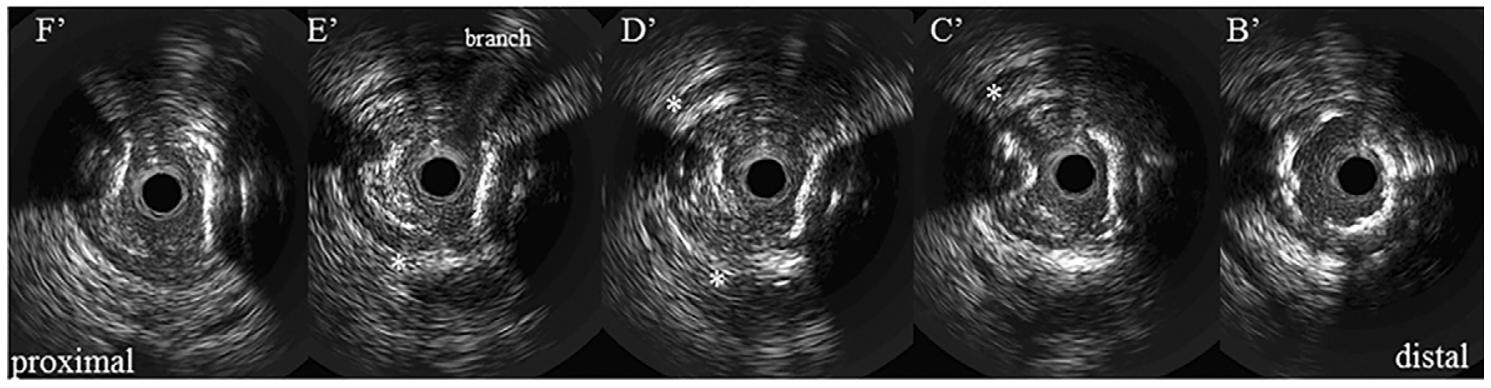

Figure 5. A: Coronary angiogram of the left circumflex artery showing subtotal occlusion. B-F: Post-procedural intravascular ultrasound (IVUS) images at the index intervention showing proximal edge dissection that reached the media. B'-F': IVUS images at the stent thrombosis corresponding to baseline IVUS images B-F showing unhealed dissection with perivascular damage (asterisk). Dissection was observed in panel $F$ ' at the stent thrombosis and was not present in the corresponding IVUS image (panel $F$ at baseline), indicating longitudinal progression of edge dissection after the index procedure.

more than eight years had passed since the BMSs were initially implanted.

\section{Stent malapposition}

Late-acquired stent malapposition was far more commonly observed with DESs, particularly in SESs, than BMSs; this may have been associated with very late stent thrombosis $(12,15-17)$. All of our cases of stent malapposition (Nos. 5-7) had received SES implantation and were described as very-late stent thrombosis (average time from stent implantation $=71$ months). In two of the three cases involving stent malapposition, we confirmed late-acquired stent malapposition due to positive vessel remodeling compared with the findings at the index IVUS procedure. Indeed, IVUS studies have revealed the main mechanism of late-acquired stent malapposition to be positive vessel remodeling $(12,18,19)$. Our present findings were consistent with those of previous reports. Furthermore, pathological studies have suggested that inflammation due to a hypersensitive reaction is a mechanism of positive vessel remodeling (20-22). These reports and the findings from the present cases suggest that there should be careful long-term follow- up of lesions with late-acquired stent apposition, particularly in cases of SES use.

\section{Stent mechanical complication: Stent fracture}

The reported frequency of stent fracture in previous studies using angiographic and/or IVUS analysis is 0.8$7.7 \%$ (23) and is much higher, up to $29 \%$, by autopsy study (24). An IVUS study revealed that stent fracture was commonly observed in patients with stent thrombosis (16). Furthermore, a pathological study demonstrated an association between thrombosis and stent fracture (24), which is also supported by case reports $(25,26)$. It has been reported that the use of SESs is an independent risk factor for stent fracture, while stent fracture can occur in both BMSs and DESs (24). An IVUS study noted that complete separation is a frequent finding in SES fracture (13). In addition to SES use, longer stent length and overlapping stents are also recognized as contributors to stent fracture (24). Our case (No. 8) well illustrates these characteristics of SES fracture leading to stent thrombosis. 


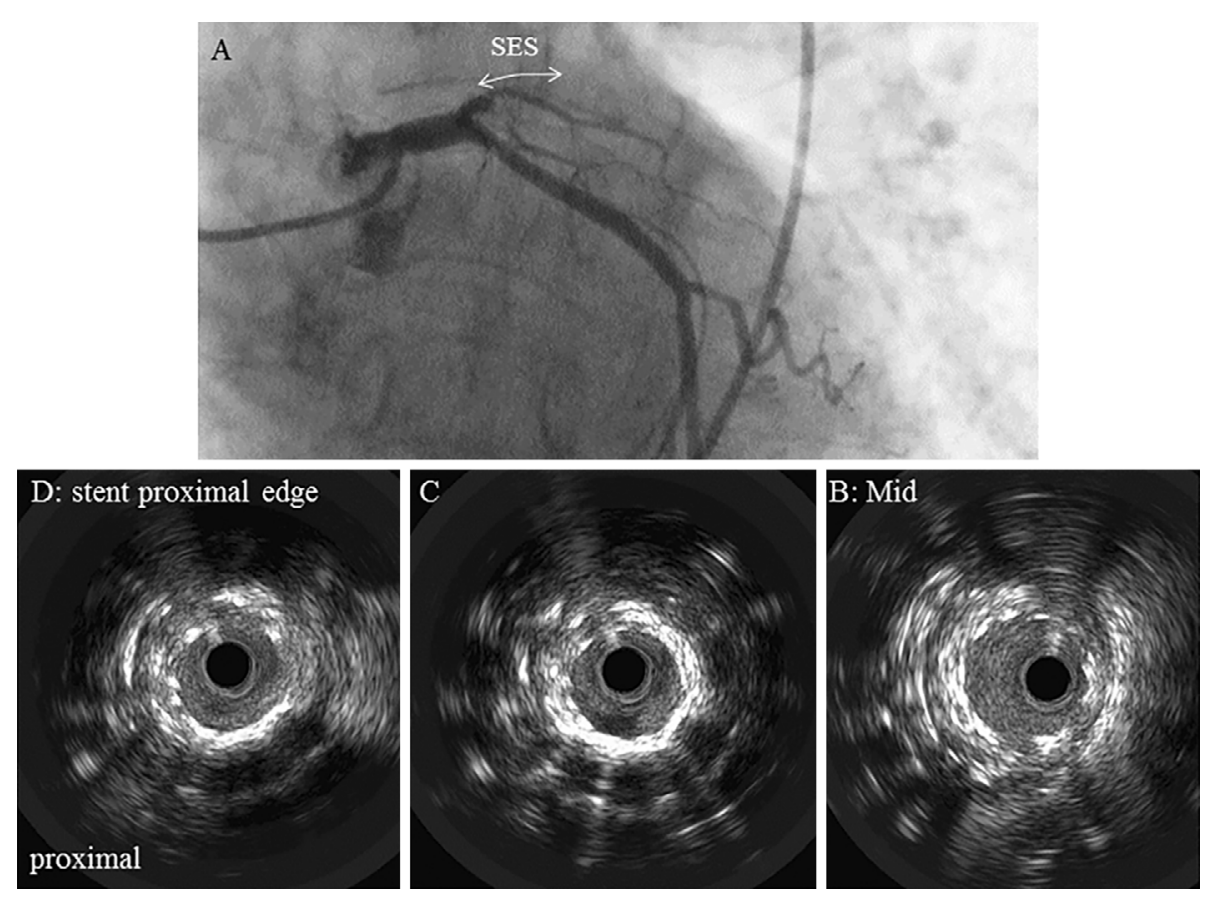

Figure 6. A: Coronary angiogram of the left anterior descending coronary artery showing total occlusion. B-D: Intravascular ultrasound images showing stent underexpansion (the minimum stent area in panel $\mathrm{C}$ was $3.8 \mathrm{~mm}^{2}$ ) without significant neointimal hyperplasia.

\section{Procedure-related complications}

Persistent dissections and slow flow, thrombus, stent underexpansion, tissue prolapse, and residual stenosis have been suggested as procedure-related contributing factors to stent thrombosis (27-30). Of these factors, we experienced persistent dissection and stent underexpansion.

\section{Edge dissection}

Although coronary dissection after percutaneous coronary intervention is not a rare complication, its detection and precise evaluation have an impact in a clinical setting, as it has been reported that coronary dissections are associated with adverse cardiovascular events $(30,31)$. A pathological study suggested that exposure of connective tissue to blood speckle in the lumen due to dissection may result in the progression of thrombus formation (32). Coronary dissections can cause perivascular trauma, including hematoma, and subsequently flow-limiting obstruction (33-35). Furthermore, previous IVUS studies have demonstrated an association between edge dissection and early stent thrombosis $(27,30)$. In our case (No.9), persistent dissection that reached the media at the proximal stent edge was observed in postprocedure IVUS of index percutaneous coronary intervention. IVUS at the stent thrombosis revealed longitudinal progression of the dissection and perivascular damage, suggesting communication between the lumen and the perivascular space. Previous reports may help clarify why stent thrombosis developed in this case. Interestingly, in this case, stent thrombosis occurred at 6.6 months after stent implantation, indicating "late" stent thrombosis. The findings from the present case suggested that longitudinal progression of the dissection might be the cause of late stent thrombosis.

\section{Chronic stent underexpansion}

Studies have demonstrated that stent underexpansion commonly contributes to BMS restenosis and that the main mechanisms of restenosis were chronic stent underexpansion and neointimal hyperplasia in the DES era $(36,37)$. In addition, Fujii et al. demonstrated that stent underexpansion was one of the independent predictors of stent thrombosis (38). Thus, it was recognized that stent underexpansion contributed to stent failure, restenosis, and stent thrombosis. However, Liu et al. suggested that underexpansion related to stent thrombosis was more severe and occurred at a more proximal location than that related to restenosis (39). The findings from our case (No. 10) were consistent with the previous report. In this case, by comparing the IVUS findings at the index procedure and at stent thrombosis, we confirmed chronic stent underexpansion without significant proliferation of neointimal hyperplasia. We therefore concluded from IVUS that the probable cause of stent thrombosis in this case was chronic severe stent underexpansion.

\section{Limitations}

The present study had several limitations. A major limitation was that it involved a retrospective analysis, so the frequency of stent thrombosis could not be determined, and the number of stent thrombosis cases was small. Because of its resolution, grayscale IVUS is limited in characterizing neointimal hyperplasia compared with optical coherence tomography. In one of three cases with stent malapposition, 

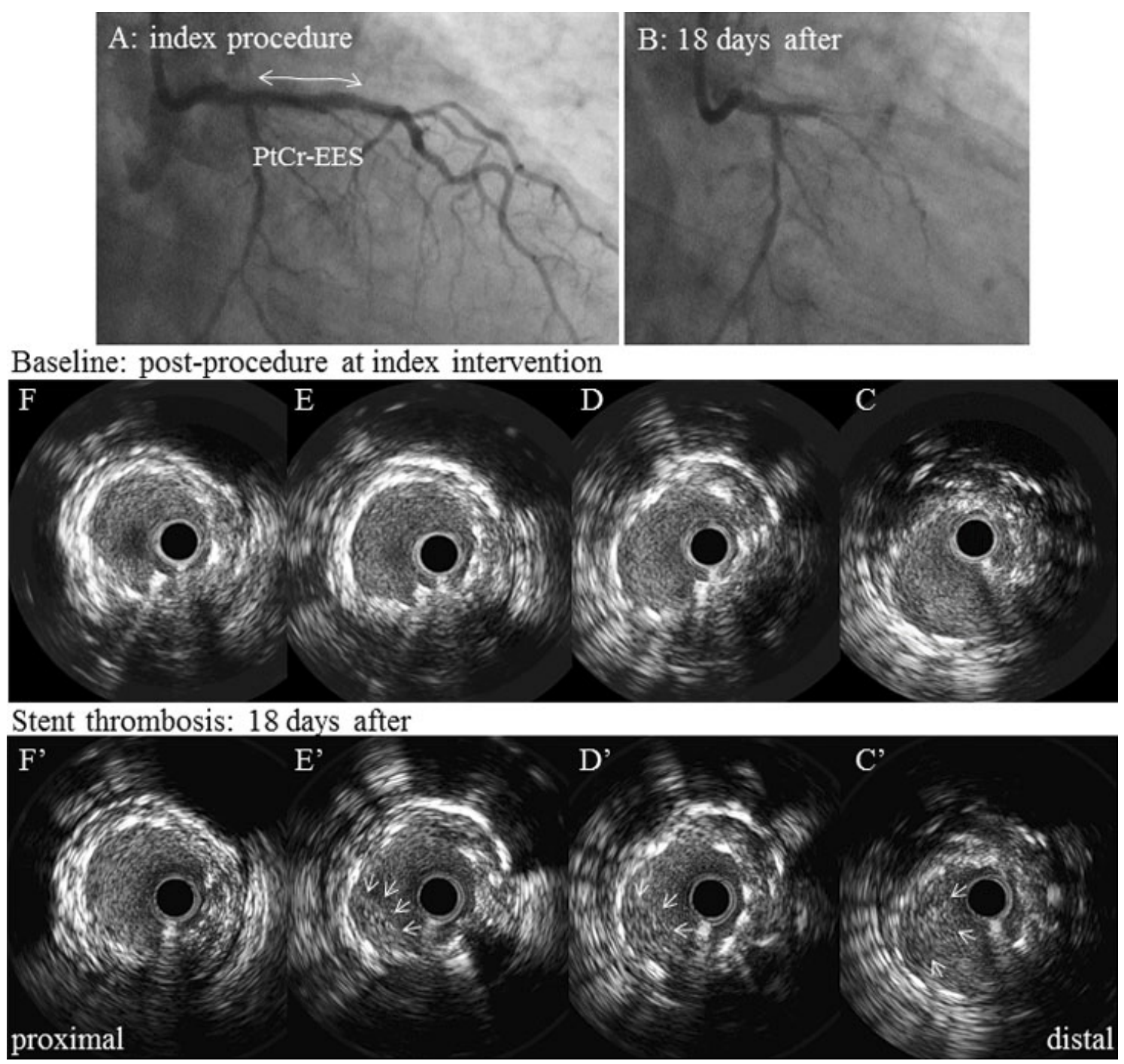

Figure 7. A: Coronary angiogram of the left anterior descending coronary artery at the index procedure. B: Coronary angiogram 18 days after the index procedure showing total occlusion. C'-F': Intravascular ultrasound (IVUS) images at the stent thrombosis showing thrombus-like appearances (white arrows); no other cause of stent failure was revealed by IVUS, compared with baseline IVUS images (C-F, corresponding to follow-up C'-F').

baseline procedural IVUS was not conducted; therefore, we were unable to conclude the cause of stent thrombosis: acute stent malapposition during the index procedure or lateacquired stent malapposition.

\section{Conclusion}

We experienced 12 definite stent thrombosis cases and evaluated the possible causes of stent thrombosis from IVUS observations. In patients with stent thrombosis, heterogeneous findings were observed in IVUS, especially in DEStreated lesions. Complex plaque morphology (rupture and malapposition) might have contributed to the occurrence of stent thrombosis. IVUS imaging insights from this case series regarding the development of stent thrombosis provide reliable information for understanding the underlying mechanisms and may help to determine a treatment strategy.
The authors state that they have no Conflict of Interest (COI).

\section{References}

1. Moses JW, Leon MB, Popma JJ, et al; SIRIUS Investigators. Sirolimus-eluting stents versus standard stents in patients with stenosis in a native coronary artery. N Engl J Med 349: 13151323, 2003.

2. Curfman GD, Morrissey S, Jarcho JA, Drazen JM. Drug-eluting coronary stents-promise and uncertainty. N Engl J Med 356: 10591060, 2007.

3. Jeremias A, Sylvia B, Bridges J, et al. Stent thrombosis after successful sirolimus-eluting stent implantation. Circulation 109: 19301932, 2004.

4. Iakovou I, Schmidt T, Bonizzoni E, et al. Incidence, predictors, and outcome of thrombosis after successful implantation of drugeluting stents. JAMA 293: 2126-2130, 2005.

5. Moreno R, Fernandez C, Hernandez R, et al. Drug-eluting stent thrombosis: results from a pooled analysis including 10 randomized studies. J Am Coll Cardiol 45: 954-959, 2005. 
6. Williams DO, Abbott JD, Kip KE; DEScover Investigators. Outcomes of 6906 patients undergoing percutaneous coronary intervention in the era of drug-eluting stents: report of the DEScover Registry. Circulation 114: 2154-2162, 2006.

7. Mintz GS. Clinical utility of intravascular imaging and physiology in coronary artery disease. J Am Coll Cardiol 64: 207-222, 2014.

8. Nakazawa G, Otsuka F, Nakano M, et al. The pathology of neoatherosclerosis in human coronary implants bare-metal and drug-eluting stents. J Am Coll Cardiol 57: 1314-1322, 2011.

9. Nakazawa G. Stent thrombosis of drug eluting stent: pathological perspective. J Cardiol 58: 84-91, 2011.

10. Cutlip DE, Windecker S, Mehran R, et al; Academic Research Consortium. Clinical end points in coronary stent trials: a case for standardized definitions. Circulation 115: 2344-2351, 2007.

11. Maehara A, Mintz GS, Bui AB, et al. Morphologic and angiographic features of coronary plaque rupture detected by intravascular ultrasound. J Am Coll Cardiol 40: 904-910, 2002.

12. Guo N, Maehara A, Mintz GS, et al. Incidence, mechanisms, predictors, and clinical impact of acute and late stent malapposition after primary intervention in patients with acute myocardial infarction: an intravascular ultrasound substudy of the Harmonizing Outcomes with Revascularization and Stents in Acute Myocardial Infarction (HORIZONS-AMI) trial. Circulation 122: 1077-1084, 2010.

13. Doi H, Maehara A, Mintz GS, et al. Classification and potential mechanisms of intravascular ultrasound patterns of stent fracture. Am J Cardiol 103: 818-823, 2009.

14. Kang SJ, Lee CW, Song H, et al. OCT analysis in patients with very late stent thrombosis. J Am Coll Cardiol Img 6: 695-703, 2013.

15. Lee CW, Kang SJ, Park DW, et al. Intravascular ultrasound findings in patients with very late stent thrombosis after either drugeluting or bare-metal stent implantation. J Am Coll Cardiol 55: 1936-1942, 2010.

16. Kosonen P, Vikman S, Jensen LO, et al. Intravascular ultrasound assessed incomplete stent apposition and stent fracture in stent thrombosis after bare metal versus drug-eluting stent treatment the Nordic Intravascular Ultrasound Study (NIVUS). Int J Cardiol 168: 1010-1016, 2013.

17. Ikenaga H, Ishihara M, Dai K, Nakama Y, Ohtani T. Mechanisms of very late stent thrombosis after drug-eluting stent implantation: findings from coronary angioscopy and optical coherence tomography. J Am Coll Cardiol Img 4: 1217-1219, 2011.

18. Shah VM, Mintz GS, Apple S, Weissman NJ. Background incidence of late apposition after bare-metal stent implantation. Circulation 106: 1753-1755, 2002.

19. Kang SJ, Mintz GS, Park DW, et al. Late and very late drugeluting stent malapposition: serial 2-year quantitative IVUS analysis. Circ Cardiovasc Interv 3: 335-340, 2010.

20. Cook S, Ladich E, Nakazawa G, et al. Correlation of intravascular ultrasound findings with histopathological analysis of thrombus aspirates in patients with very late drug-eluting stent thrombosis. Circulation 120: 391-399, 2009.

21. Finn AV, Nakazawa G, Joner M, et al. Vascular responses to drug eluting stents: importance of delayed healing. Arterioscler Thromb Vasc Biol 27: 1500-1510, 2007.

22. Virmani R, Guagliumi G, Farb A, et al. Localized hypersensitivity and late coronary thrombosis secondary to a sirolimus-eluting stent: should we be cautious? Circulation 109: 701-705, 2004.

23. Inaba S, Mintz GS, Yun KH, et al. Mechanical complications of everolimus-eluting stents associated with adverse events: an intravascular ultrasound study. Eurointervention 9: 1301-1308, 2014.

24. Nakazawa G, Finn AV, Vorpahl M, et al. Incidence and predictors of drug-eluting stent fracture in human coronary artery a pathologic analysis. J Am Coll Cardiol 54: 1924-1931, 2009.

25. Shite J, Matsumoto D, Yokoyama M. Sirolimus-eluting stent fracture with thrombus, visualization by optical coherence tomography. Eur Heart J 27: 1389, 2006.

26. Leong DP, Dundon BK, Puri R, Yeend RA. Very late fracture associated with a sirolimus-eluting stent. Heart Lung Circ 17: 426428, 2008.

27. Cheneau E, Leborgne L, Mintz GS, et al. Predictors of subacute stent thrombosis: results of a systematic intravascular ultrasound study. Circulation 108: 43-47, 2003.

28. Moussa I, Di Mario C, Reimers B, Akiyama T, Tobis J, Colombo A. Subacute stent thrombosis in the era of intravascular ultrasound-guided coronary stenting without anticoagulation: frequency, predictors and clinical outcome. J Am Coll Cardiol 29: 612, 1997.

29. Uren NG, Schwarzacher SP, Metz JA, et al; POST Registry Investigators. Predictors and outcomes of stent thrombosis: an intravascular ultrasound registry. Eur Heart J 23: 124-132, 2002.

30. Choi SY, Witzenbichler B, Maehara A, et al. Intravascular ultrasound findings of early stent thrombosis after primary percutaneous intervention in acute myocardial infarction: a Harmonizing Outcomes with Revascularization and Stents in Acute Myocardial Infarction (HORIZONS-AMI) substudy. Circ Cardiovasc Interv 4: 239-247, 2011.

31. Biondi-Zoccai GG, Agostoni P, Sangiorgi GM, et al; Real-world Eluting-stent Comparative Italian retrosPective Evaluation Study Investigators. Incidence, predictors, and outcomes of coronary dissections left untreated after drug-eluting stent implantation. Eur Heart J 27: 540-546, 2006.

32. Block PC, Myler RK, Stertzer S, Fallon JT. Morphology after transluminal angioplasty in human beings. $\mathrm{N}$ Engl J Med 305: 382-385, 1981.

33. Maehara A, Mintz GS, Bui AB, et al. Incidence, morphology, angiographical findings, and outcomes of intramural hematomas after percutaneous coronary interventions: an intravascular ultrasound study. Circulation 105: 2037-2042, 2002.

34. Maehara A, Mintz GS, Bui AB, et al. Intravascular ultrasound evidence of perivascular trauma during routine percutaneous coronary intervention. Int J Cardiovasc Imaging 30: 849-856, 2014.

35. Inaba S, Mintz GS, Collins MB, Fall KN, Moses JW, Maehara A. Acute closure due to extramedial hematoma 3 hours after stenting. J Am Coll Cardiol Interv 7: e19-e21, 2014.

36. de Feyter PJ, Kay P, Disco C, Serruys PW. Reference chart derived from post-stent-implantation intravascular ultrasound predictors of 6-month expected restenosis on quantitative coronary angiography. Circulation 100: 1777-1783, 1999.

37. Kang SJ, Mintz GS, Park DW, et al. Mechanisms of in-stent restenosis after drug-eluting stent implantation: intravascular ultrasound analysis. Circ Cardiavasc Interv 4: 9-14, 2011.

38. Fujii K, Carlier SG, Mintz GS, et al. Stent underexpansion and residual reference segment stenosis are related to stent thrombosis after sirolimus-eluting stent implantation: an intravascular ultrasound study. J Am Coll Cardiol 45: 995-998, 2005.

39. Liu X, Doi H, Maehara A, et al. A volumetric intravascular comparison of early drug-eluting stent thrombosis versus restenosis. J Am Coll Cardiol Interv 2: 428-434, 2009.

The Internal Medicine is an Open Access article distributed under the Creative Commons Attribution-NonCommercial-NoDerivatives 4.0 International License. To view the details of this license, please visit (https://creativecommons.org/licenses/ by-nc-nd/4.0/).

(C) 2017 The Japanese Society of Internal Medicine

http://www.naika.or.jp/imonline/index.html 